Original Research Article

\title{
Evaluation and comparison of the hepatoprotective effects of trimetazidine and lovastatin against doxorubicin-induced hepatotoxicity
}

\author{
Abeera Sikandar ${ }^{*}$, Kulsoom Farhat ${ }^{2}$, Amir Hamza ${ }^{3}$
}

\begin{abstract}
${ }^{1}$ Department of Pharmacology, Wah Medical College, National University of Medical Sciences (NUMS), Rawalpindi, Pakistan ${ }^{2}$ Department of Pharmacology, Army Medical College, National University of Medical Sciences (NUMS), Rawalpindi, Pakistan ${ }^{3}$ Department of Mechatronics Engineering, National University of Science and Technology (NUST), Rawalpindi, Pakistan
\end{abstract}

Received: 16 January 2019 Accepted: 27 February 2019

*Correspondence to: Dr. Abeera Sikandar, Email: abeera.sikandar@ gmail.com

Copyright: (C) the author(s), publisher and licensee Medip Academy. This is an openaccess article distributed under the terms of the Creative Commons Attribution NonCommercial License, which permits unrestricted noncommercial use, distribution, and reproduction in any medium, provided the original work is properly cited.

\begin{abstract}
Background: The dosage of highly efficacious anti-cancer drug doxorubicin (DOX) is often constrained as limited data exists on its hepatotoxic potential. The present study not only evaluated the extent of its hepatotoxicity but also aimed at curtailing it, by administration of two drugs i.e. trimetazidine and lovastatin, both of which are otherwise known for their cardioprotective benefits.

Methods: The study was a lab-based randomized controlled study on mice. Acute toxicity was introduced with DOX injected intraperitoneally at a dose of 10 $\mathrm{mg} / \mathrm{kg}$ and it was protected by oral administration of trimetazidine and lovastatin, both in a dose of $10 \mathrm{mg} / \mathrm{kg}$. The protective drugs were both given for five and ten consecutive days in short and long term study designs whereby DOX was administered on the third and eighth days of the respective studies.

Results: Doxorubicin administration caused significant hepatotoxicity reflected by markedly raised biomarkers (serum alanine aminotransferase and aspartate aminotransferase) and mild inflammation of liver parenchyma with a score of 4 as per Ishak grading scale. The changes were significantly attenuated by both the protective drugs in the ten days study design. However, in the five days study, lovastatin exhibited more significant hepatoprotection than trimetazidine.

Conclusions: Pretreatment with two commonly available, cost effective and safe drugs can effectively prevent a potentially life-threatening adverse effect of DOX. This approach might prove very convenient for the health care providers as well as for the patients without burdening the economics.
\end{abstract}

Keywords: Alanine aminotransferase, Aspartate aminotransferase, Doxorubicin, Hepatotoxicity, Lovastatin, Trimetazidine

\section{INTRODUCTION}

Doxorubicin (DOX), a derivative of Streptomyces peucetius, is one of the most broad spectrum anthracycline antineoplastic agents used in a large number of solid and hematological malignancies. ${ }^{1}$ Due to the dose related organ damage associated with its use, it is advised to limit the dosage to 450 to $550 \mathrm{mg} / \mathrm{m}^{2} .^{2}$ Although heart is the most commonly affected organ, but liver, being the main detoxifying organ, has also been reported in various experimental animal studies. ${ }^{3,4}$ Regarding the human data, Zhao et al, reported it to be $40 \%$; and the incidence was concluded to be $30.4 \%$ in another study on breast cancer patients that received $\mathrm{DOX}^{5,6}$ The most probable 
mechanisms for the DOX hepatotoxicity includes topoisomerase II inhibition leading to arrest of the cell cycle of hepatocytes and reactive oxygen species (ROS) generation. ${ }^{7}$ The accumulation of the cytotoxic DOX due to hepatic injury also puts a threat of augmenting the very common phenomenon of cardiotoxicity associated with DOX. It's therefore crucial to give hepatotoxicity due consideration while designing the dose and to introduce cost effective pharmacological modulations that would supplement its chemotherapeutic benefit besides curtailing its toxicity. ${ }^{8}$

Till date, several pharmacologic agents with potent antioxidants properties have been tested against the hepatotoxicity of DOX. ${ }^{3,7,9}$ Although most of these agents were confirmed to be beneficial in the animal studies, their extrapolation in clinical trials has been implausible and none is FDA approved till date. Trimetazidine (TMZ) is an effective anti-anginal agent that improves the contractile response of chronically dysfunctional myocardium. It is shown to have remarkable cytoprotective and antioxidant properties. TMZ prevents beta-oxidation of fatty acids thus promoting glucose oxidation and preserving cellular energy. ${ }^{10,11}$ Widely known for cardio protection, it is also being studied for its hepatoprotective benefits against various chemotherapeutic drugs including DOX. ${ }^{2,12,13}$ Its hepatoprotective benefits in different doses and schedules for diverse causes of hepatic injuries have been studied. ${ }^{13,14}$ This study was especially formulated to reach at an optimal pre-treatment dose and duration for TMZ to counter oxidative stress due to DOX.

Lovastatin, the other protective agent used in the study, is primarily a lipid lowering drug that blocks the enzyme (3hydroxy-3-methyl-glutaryl-coenzyme A) HMG-CoA reductase causing reduced levels of cholesterol. In recent times, substantial data has shown that statins exert numerous effects unrelated to their plasma cholesterol lowering properties. ${ }^{15}$ They are documented to have potent antioxidant and anti-inflammatory properties. ${ }^{16,17}$ Lovastatin has been reported to exhibit antitumor effects in rodent tumor models and to reinforce the antitumor effects of various anti - cancer drugs. ${ }^{18,19}$ Keeping in view the pleiotropic effects, researchers have suggested that it could prevent DOX induced pro inflammatory and pro fibrotic stress responses. ${ }^{16,19-21}$

Considering the high incidence of disproportionate adverse effects of anti-cancer drugs, only time tested agents like DOX would be preferred for a long time. Co administration of drugs with cytoprotective potential and safe adverse effect profile is integral for ensuring safer chemotherapy. This study is a part of the continued spectrum of research on toxicity of anti-neoplastic drugs and exploration of better and safer protective agents. Although TMZ and lovastatin have been shown to attenuate DOX induced toxicity, ${ }^{2,16,19,21}$ but most of these study designs were inadequate to make consistent conclusions for clinical translation. Henceforth, this study was particularly designed to evaluate and compare the hepatoprotective potential of two diverse drugs in two different pre-treatment schedules to encourage feasible clinical trials.

\section{METHODS}

\section{Drugs and experimental setting}

The study was carried out in the animal house of Department of Pharmacology and Therapeutics, Army medical College (AMC), Rawalpindi. DOX HCL (Adriamycin) was arranged from Bone $\mathrm{M}$ arrow and Transplant Centre (BMTC), Rawalpindi while lovastatin and TMZ were purchased from the local pharmacies. Study protocol was approved by the Ethical Committee of Centre for Research in Experimental and Applied Medicine (CREAM), Army Medical College. The biochemical and histopathological examinations were performed in collaboration with the Departments of Chemical Pathology and Histopathology, AMC.

$64 \mathrm{Balb} / \mathrm{c}$ mice of age 8-12 weeks and weight $40 \pm 5$ grams were procured from National Institute of Health (NIH), Islamabad and were acclimatized for a week before the start of experiment. They were kept under standard laboratory conditions of 12 hours light and dark cycle, $20 \pm 25^{\circ} \mathrm{C}$ temperature and $70 \pm 15$ percent humidity. Mice were given the rodent pellet diet and tap water ad libitum throughout the span of experiment.

\section{Experimental design}

The study was a laboratory based randomized controlled trial; animals were chosen by non- probability convenience sampling method and randomly allocated into eight groups $(n=8)$. The span of study for group 1 to 6 was five days while that for group 7 and 8 was ten days.

\section{Groups 1 to 6}

Group one served as the control group. Group two mice were injected DOX intraperitoneally (IP) at a dose of 10 $\mathrm{mg} / \mathrm{kg}$ on the $3^{\text {rd }}$ day of experiment. Groups three and four mice received the protective drugs i.e. TMZ and lovastatin orally both in a dose of $10 \mathrm{mg} / \mathrm{kg}$ for five consecutive days. Groups five and six received the protective drugs for five consecutive days and DOX was administered on the 3rd day of experiment. Animals in these groups were sacrificed on day five.

\section{Group 7 and 8}

Animals in groups 7 and 8 received TMZ and lovastatin respectively in an oral dose of $10 \mathrm{mg} / \mathrm{kg}$ for ten consecutive days and received DOX intraperitoneally at a dose of $10 \mathrm{mg} / \mathrm{Kg}$ on the $8^{\text {th }}$ day. Animals were sacrificed on day ten of the study.

Mice were euthanized with a humane approach as per the protocol of Animal Welfare Act and Animal Welfare 
Regulation, 2013. Ether was used for the initiation of anesthesia using drop jar method.

\section{Biochemical analysis}

Blood samples were collected terminally by intra cardiac puncture for the analysis of Alanine aminotransferase (ALT) and Aspartate aminotransferase (AST). ALT was estimated by kinetic method while AST was estimated by optimized UV method. Both were performed using kits manufactured by Diasys Diagnostic System USA on automated chemistry analyzer SELECTRA E according to the principles laid down by International Federation of Clinical Chemistry (IFCC).

\section{Histopathological analysis}

After the terminal blood sampling, liver was dissected out and was mixed immediately in $10 \%$ phosphate buffered formaldehyde for fixation. All the samples were later dried in increasing alcohol concentrations, cleared with xylene and imbued in paraffin after which they were cut into four micrometer thin sections by a rotatory microtome. They were stained with Hematoxylin and Eosin $(\mathrm{H} \& \mathrm{E})$ dyes and were examined thoroughly under light microscope. Ishak Histological Activity Index (HAI) was used to grade histopathological changes in the liver.

\section{Statistical analysis}

It was done on SPSS version 22. Results were expressed as Mean \pm Standard Error of Mean (S.E.M). One way analysis of variance (ANOVA) and Post Hoc Tukey Test were used for multiple comparisons of biochemical markers between the groups. Histopathological findings were analyzed by Chi Square Test. The difference between two observations was considered significant if the $p$ value was less than 0.05 .

\section{RESULTS}

\section{Serum ALT}

Dox administration in group 2 led to marked elevation ( $\mathrm{p}$ value $\leq 0.0001$ ) of ALT with a mean of $516.87 \pm 74.48$ against $36.25 \pm 5.15$ in the control group. The five days administration of trimetazidine (group 5) led to nonsignificant reduction. However, extending the treatment duration to ten days in group 7 significantly prevented the elevation in the ALT levels ( $\mathrm{p}$ value $\leq 0.05$ ) (Table 1 and Figure 1). The administration of lovastatin both for three and eight days prior to Dox in group 6 and 8 led to highly significant reduction in the enzyme levels with the means of $185.12 \pm 36.02$ and $107.12 \pm 16.09$ respectively with a $p$ value $\leq 0.0001$ (Table 2 and Figure 2).

Table 1: Effect of TMZ administration for 5 and 10 days on the biochemical parameters of DOX treated mice.

\begin{tabular}{|llllll||}
\hline & Control & TMZ & DOX & DOX/TMZ-5d & DOX/TMZ-10d \\
\hline ALT & $36.25 \pm 5.15$ & $43.75 \pm 3.24$ & $516.87 \pm 74.48^{\#}$ & $344.75 \pm 66.65$ & $183.0 \pm 53.65^{*}$ \\
\hline AST & $126.25 \pm 17.78$ & $182.5 \pm 13.85$ & $798.12 \pm 55.34^{\#}$ & $518.12 \pm 68.29^{*}$ & $223.5 \pm 28.29^{* *}$ \\
\hline
\end{tabular}

\#Signifiant results compared to control ( $\mathrm{p}$ value $\leq 0.0001$ ), *Significant results compared to DOX ( $\mathrm{p}$ value $\leq 0.05$ ); $* *$ Highly significant results compared to DOX ( $\mathrm{p}$ value $\leq 0.0001$ ).

Table 2: Effect of lovastatin administration for 5 and 10 days on the biochemical markers of DOX treated mice.

\begin{tabular}{|llllll|}
\hline & Control & Lovastatin & DOX & DOX/Lova -5d & DOX/Lova-10d \\
\hline ALT & $36.25 \pm 5.15$ & $46.75 \pm 4.2$ & $516.875 \pm 74.48^{\#}$ & $185.12 \pm 36.02^{* *}$ & $107.12 \pm 16.69^{* *}$ \\
\hline AST & $126.25 \pm 17.78$ & $133.5 \pm 11.04$ & $798.125 \pm 55.34^{\#}$ & $314.12 \pm 43.63^{* *}$ & $179.25 \pm 13.0^{* *}$ \\
\hline
\end{tabular}

\#Signifiant results compared to control ( $\mathrm{p}$ value $\leq 0.0001$ ); **Highly significant results compared to DOX ( $\mathrm{p}$ value $\leq 0.0001$ ).

\section{Histological examination}

DOX induced hepatic damage is described by scattered areas of inflammatory cellular infiltration, venous congestion, dilated sinusoids, portal and peri-portal inflammation. The changes were assessed to be mild with a score of 4 as per the ishak HAI scale (Figure 3B). As the toxic dose was single, we did not see any chronic inflammatory changes.

Treatment of mice with TMZ for five days slightly prevented DOX induced pathological changes as focal inflammation and sinusoid dilation was still seen in 6 slides
(Figure 3C). Lovastatin for five days efficiently prevented the architecture as minimal periportal inflammation was seen in 5 slides of this group ( Figure 3E). The ten days administration by TMZ led to a remarkable protection as 5 out of 8 slides were graded to be normal while all the slides were graded to be normal after the ten days administration of lovastatin (Figures 3D and 3F).

\section{Serum AST}

The administration of Dox also raised the level of AST significantly ( $p$ value $\leq 0.0001$ ) to a value of $798.12 \pm 55.34$ against $126.25 \pm 17.78$ in group 1 . 


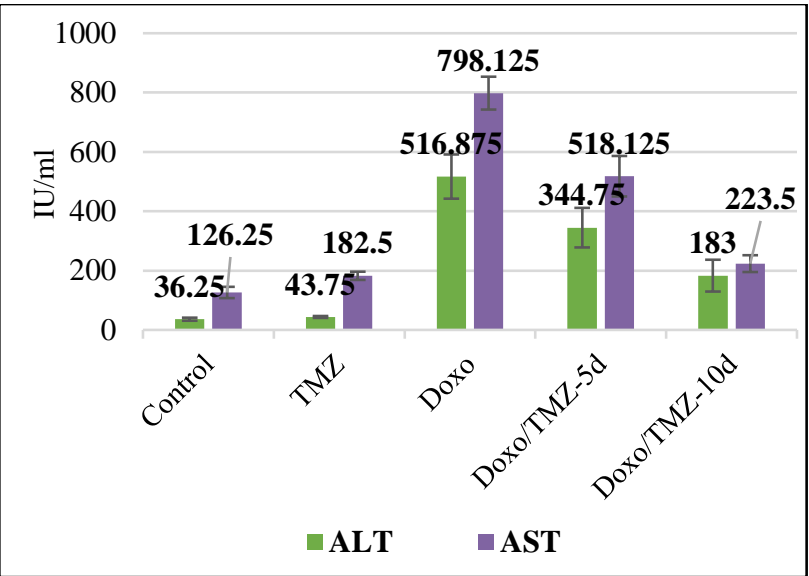

Figure 1: The effects of TMZ administration for two different durations on the hepatic biochemical markers of DOX treated animals.

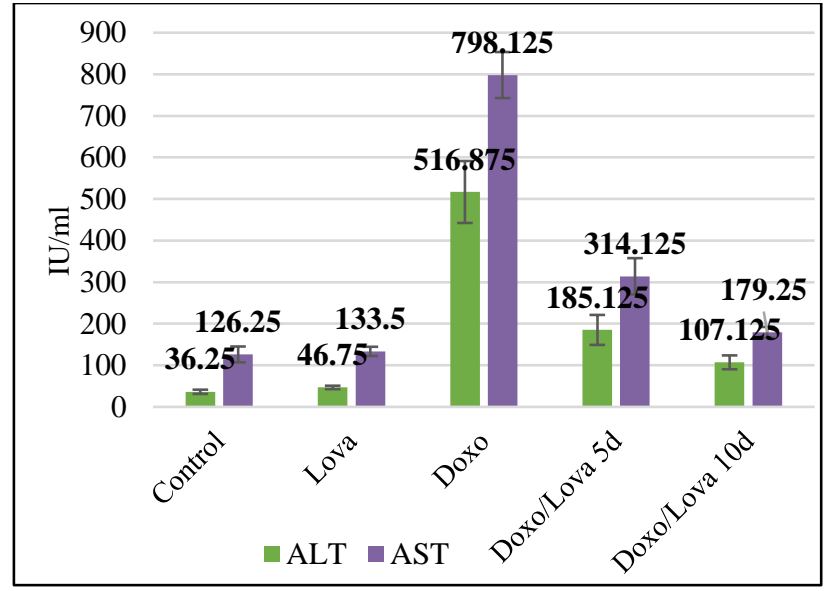

Figure 2: The effects of TMZ administration for two different durations on the biochemical markers of DOX treated animals.

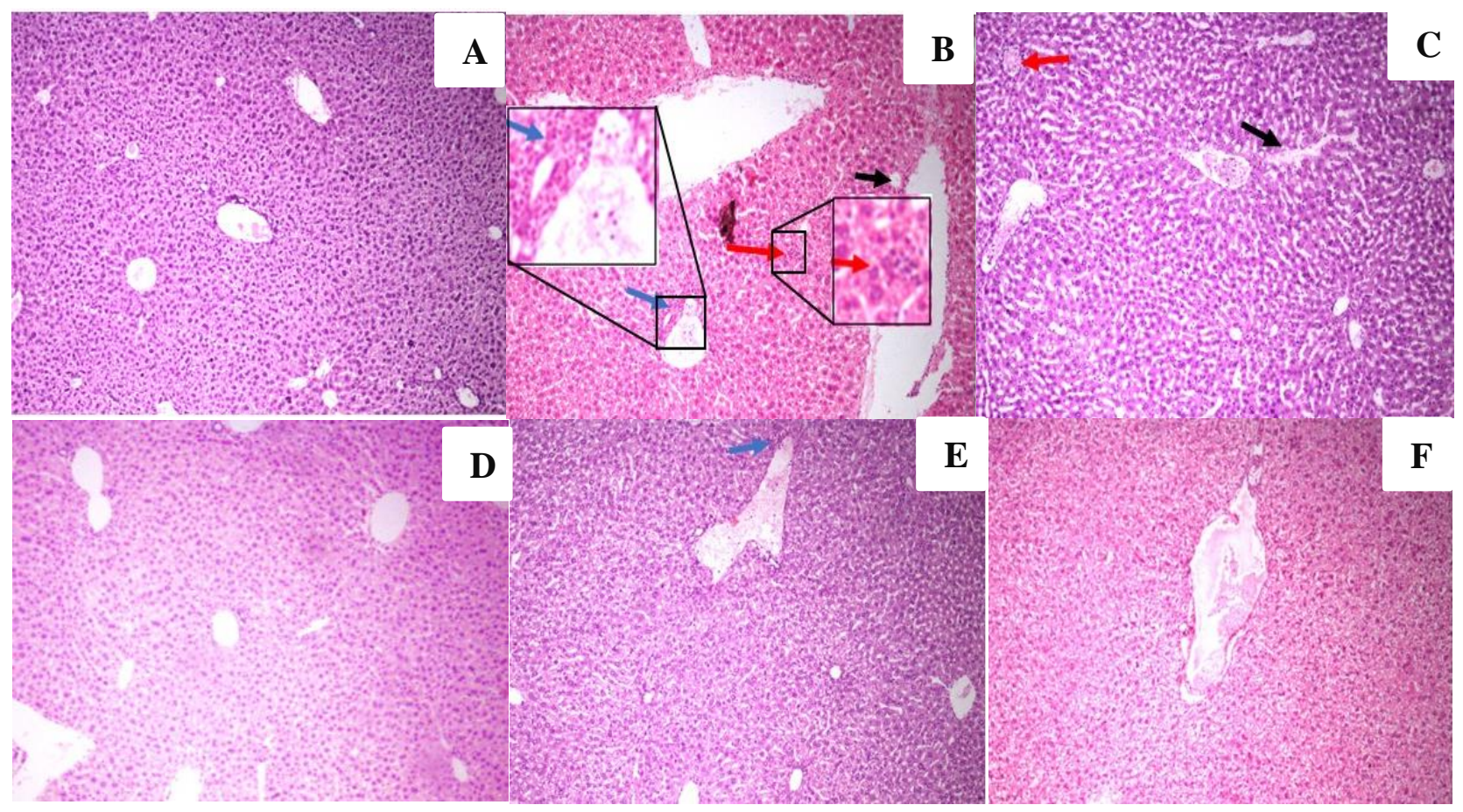

Figure 3: Photomicrograph of liver tissues (H\&E 100x): (A): group 1 (control) with normal hepatic parenchyma, (B): group 2 (DOX) with focal inflammation (red arrow), portal inflammation (blue arrow) and dilated sinusoids (black arrow), (C): group 5 (DOX+TMZ5d) with minimal focal inflammation and dilated sinusoids, (D): group 7

(DOX+TMZ10d) with normal parenchyma, (E): group 6 (DOX+Lova5d) with minimal peri portal inflammation, (F): group 8 (DOX+Lova10d) with almost restored architecture.

The administration of trimetazidine for three days significantly prevented the elevation ( $\mathrm{p}$ value $\leq 0.05$ ) and the prevention was even more effective in group 7 ( $\mathrm{p}$ value $\leq 0.0001$ ) (Table 1 and Figure 1). In groups 6 and 8 , lovastatin effectively prevented the enzyme surge ( $p$ value $\leq 0.0001$ ) with values of $314.12 \pm 43.63$ and $179.25 \pm 13.0$ respectively (Table 2 and Figure 2).

\section{DISCUSSION}

The experimental data available on hepatoprotection against doxorubicin (DOX) is still less compared to that on cardioprotection. ${ }^{22}$ However, the prevalence of hepatic disorders hinders the successful use of DOX and limits the oncologists from achieving the maximum benefit of this 
potent drug..$^{3,7,23}$ The injury to liver is especially pertinent keeping in view the liver's key role in metabolism of DOX and is reported to occur even in the therapeutic doses. ${ }^{5,12}$ This study was designed with an aim of comparing the hepatoprotection by trimetazidine (TMZ) and lovastatin, both commonly known for their cardioprotective benefits, in two different pre-treatment schedules. The drugs were also administered alone in two groups to establish that they themselves did not affect the liver enzymes.

Hepatotoxicity was produced with a single $10 \mathrm{mg} / \mathrm{kg}$ intraperitoneal injection of DOX as ALT and AST were increased significantly ( $p$ value $\leq 0.0001$ ) compared with the control. These findings corresponded to the deteriorated histological findings on microscopic examination which were categorized as mild per ISHAK HAI (histologically active index). The toxicity seen was in accordance with many previous studies. ${ }^{2-5,7,19}$ The serum transaminases are sensitive markers of liver injury. ${ }^{8}$ Especially, ALT is a highly specific diagnostic marker of liver parenchymal injury. Histopathological changes in liver, however, were observed to be minimal to mild and were calculated to be statistically significant compared with the control. The highly significant enzyme elevation was not reflected in the microscopic analysis as it takes longer for the histological changes to appear. Morphological changes in liver due to DOX have also been observed in various animal studies. ${ }^{4,9}$

The mechanism of DOX hepatotoxicity includes the inhibition of topoisomerase II causing interference with DNA unwinding and subsequent inhibition of DNA replication. ${ }^{24}$ Generation of oxygen free radicals is also a crucial cause of hepatocyte fragility and enzyme release. DOX also stimulates phospholipases which raise intracellular calcium and lead to ALT release from apoptotic hepatocytes. ${ }^{4}$ It also modifies the defense mechanism leading to a decreased detoxification of ROS. ${ }^{25}$ Co administering drugs with antioxidants properties is therefore estimated to be the desirable protective approach.

In two of the groups, we investigated the hepatoprotective role of TMZ, a cytoprotective drug used in angina patients and has been remarkably successful in reducing the attacks. ${ }^{10,11,26}$ In present short scheme of study (group 5) where TMZ was administered for three days prior to DOX, there was no significant attenuation of one of the two biochemical parameters i.e. ALT (33.30\% reduction only). This deficient reversion of the damage was also reflected in the microscopic examination. However, longer pretreatment for eight days in group 7 significantly reduced the enzyme upsurge for both the ALT and AST (64.59\% and $71.99 \%$ decrease respectively) as well as re-established the hepatic histopathological architecture. Present study results of group 5 were not in agreement with a short term toxic model of DOX whereby administration of TMZ with DOX for 3 days significantly mitigated its cardiotoxicity and hepatotoxicity. ${ }^{2}$ The ten day study results, however, were in consistent with another study where TMZ protection was tested against cisplatin induced hepatotoxicity. ${ }^{13}$ In another study, two protective doses i.e. 5 and $10 \mathrm{mg} / \mathrm{kg}$ of TMZ to experimental rats were given whereby extensive liver damage was introduced by carbon tetra chloride administration. Definitive improvement was exhibited at the dose of $10 \mathrm{mg} / \mathrm{kg} .{ }^{12}$ So several times and doses of administration for TMZ have been proposed in the literature. Boussaid et al, in their study on hepatic ischemic reperfusion injury have proposed $10 \mathrm{mg} / \mathrm{kg}$ per day for at least 7 consecutive days to be the optimum TMZ dosage for most effective protection at cellular and mitochondrial level. ${ }^{14}$

The result of present study revealed that it is significantly beneficial to increase the number of pre-treatment days with TMZ for effective preconditioning of hepatocytes. The hepatoprotective and anti-oxidant properties of TMZ appear multifaceted. Apart from reduction in oxidative stress, preservation of liver energy metabolism plays crucial role in cellular protection. The membrane damage is also protected by decreased intracellular acidosis and inhibited production of free radicals. ${ }^{12}$

In groups 6 and 8, lovastatin was administered in a dose of $10 \mathrm{mg} / \mathrm{kg} / \mathrm{d}$ orally for five and ten consecutive days respectively. In group 6 , the three days pre-treatment conferred significant hepatoprotection as is evident by significant decrease in both the biochemical markers i.e. ALT and AST ( $p$ value $\leq 0.0001$ ). This decreased was also supported by the relevant histological findings as fewer slides displayed the portal inflammation and sinusoidal dilation. A relatable short term study investigated the effect of lovastatin on acute hepatotoxicity of DOX and pretreated mice with a human relevant dose of lovastatin (10 $\mathrm{mg} / \mathrm{kg}$ ) before DOX administration. ${ }^{19}$ The results of this short hepatotoxic model were consistent with our short term model results. No study of lovastatin's pretreatment for a week has ever been conducted. The longer administration in group 8 was even more successful in bringing down the hepatic injury markers in comparison to group $6(79.27 \%$ decrease in ALT as compared to $64.29 \%$ in the short term administration; $77.54 \%$ decrease in AST against $60.64 \%$ decrease). The histopathological observation was also in absolute accordance with the biochemical changes. Taken together, it was shown that lovastatin protected the mice from DOX induced hepatic injury in both the schemes of study.

Lovastatin largely attenuates acute pro-inflammatory and pro-fibrotic stress response of the liver after anthracycline treatment. ${ }^{19,21}$ The mechanism of lovastatin in reverting these toxic changes is suggested to be a consequence of the lipid- independent inhibition of isoprenoids and their related molecular events. It inhibits isoprenylation of Ras and Rho G-proteins, which regulate several vital functions like cell proliferation, differentiation, and apoptosis. So, lovastatin with its antioxidant, anti-inflammatory and antiapoptotic properties turns out to be beneficial adjuvant in DOX chemotherapy. Also, many in vitro and in vivo studies propose that lovastatin is an antiangiogenic drug and could offer more benefits in cancer patients besides 
hepatoprotection. ${ }^{15,27}$ Statins, other than lovastatin, that are lipophilic are also being successfully studied for the protective benefits against DOX toxicity. ${ }^{9,28}$

It's important to know that statin administration in cancer regimen, as is the case here, would be very low in dose and duration than for their use as anti-lipid agents. They actually appear to be related to a very low risk of liver damage. For instance, it was reported that only $3 \%$ of the patients on statins against $1.1 \%$ on placebo exhibited elevation of ALT and this laboratory abnormality is often not associated with histopathological changes. ${ }^{29,30}$

\section{CONCLUSION}

Doxorubicin induced hepatotoxicity is a relevant but underexplored phenomenon. In this experimental study, it is significantly prevented by administration of two diverse drugs i.e. trimetazidine and lovastatin. While designing an optimal pre-treatment schedule for these drugs, lovastatin was concluded to be a better hepatoprotective agent offering earlier and more significant hepatoprotection. The promising results could have a substantial impact towards ensuring safer chemotherapy. The results also reflect the wide-ranging potential of statins and endorse the belief that disproportionate fear of their hepatotoxicity should not prevent exploration of their newer therapeutic indications.

\section{ACKNOWLEDGEMENTS}

Authors would like to acknowledge the financial assistance of National University of Medical Sciences (NUMS), Pakistan.

\section{Funding: No funding sources}

Conflict of interest: None declared

Ethical approval: The study was approved by the Ethical Committee of Centre for Research in Experimental and Applied medicine (CREAM), Army Medical College, Rawalpindi

\section{REFERENCES}

1. Harake D, Franco VI, Henkel JM, Miller TL, Lipshultz SE. Cardiotoxicity in childhood cancer survivors: strategies for prevention and management. Future Cardiol. 2012 Jul;8(4):647-70.

2. Salouege I, Ali RB, Saïd DB, Elkadri N, Kourda N, Lakhal M, et al. Means of evaluation and protection from doxorubicin-induced cardiotoxicity and hepatotoxicity in rats. J Cancer Res Therapeut. 2014 Apr 1;10(2):274.

3. Nagai K, Oda A, Konishi H. Theanine prevents doxorubicin-induced acute hepatotoxicity by reducing intrinsic apoptotic response. Food Chem Toxicol. 2015;78:147-52.

4. Hozayen WG, Seif HSA, Amin S. Protective Effects of Ruitn and / or Hesperidin Against DoxorubicinInduced Hepatotoxicity. Int J Clin Nutr. 2014;2(1):117.
5. Zhao X, Zhang J, Tong N, Chen Y, Luo Y. Protective effects of berberine on Doxorubicin-induced hepatotoxicity in mice. Biol Pharm Bull. 2012;35(5):796-800.

6. Damodar G, Smitha T, Gopinath S, Vijayakumar S, Rao Y. An evaluation of hepatotoxicity in breast cancer patients receiving injection Doxorubicin. Ann Med Health Sci Res. 2014 Jan;4(1):74-9.

7. Al-zubaidy AA, Khattab YI. The Possible Protective Effect of Pentoxifylline against Doxorubicin-Induced Hepatotoxicity Rabbits. 2014;2(9).

8. Thatishetty AV, Agresti N, O'Brien CB. Chemotherapy-induced hepatotoxicity. Clin Liver Dis. 2013 Nov 1;17(4):671-86.

9. Morris CA, El-Hiti GA, Weeks I, Woodhead S, Smith K, Kille P. Quantitative analysis of gene expression changes in response to genotoxic compounds. Toxicol Vitro. 2017 Mar 1;39:15-28.

10. Dézsi CA. Trimetazidine in Practice: Review of the Clinical and Experimental Evidence. Am J Ther. 2013;30(3):e871-9.

11. Tsioufis K, Andrikopoulos G, Manolis A. Trimetazidine and Cardioprotection. Angiology. 2015;66(3):204-10.

12. Mate V, Pandit V, Wani D, Dhande P. Role of trimetazidine in carbon tetrachloride induced liver damage in rats. Int $\mathrm{J}$ Basic Clin Pharmacol. 2014;3(1):164.

13. Ateyya H, Yosef H, Nader MA. Ameliorative effect of trimetazidine on cisplatin-induced hepatotoxicity in rats. Canadian J Physiol Pharmacol. 2015 Aug 19;94(2):225-30.

14. Boussaid AM, Selmi R, Bejaoui M, AYED KH, Zaouali MA, Abdennebi HB. Effectiveness of a single versus repeated administration of trimetazidine in the protection against warm ischemia/reperfusion injury of rat liver. Turkish J Med Sci. 2016 Jun 23;46(4):1258-64.

15. Rohilla A, Rohilla S, Kumar A, Khan MU, Deep A. Pleiotropic effects of statins: a boulevard to cardioprotection. Arabian J Chem. 2016 Sep 1;9:S217.

16. Henninger C, Ohlig J, Bopp A, Wartlick F, Merx M, Fritz G. Targeting of RAC1-signaling for the prevention of anthracycline-induced cardio-and hepatotoxicity. Support Care Cancer. 2014;22(1):S161.

17. Kumar S, Srivastava N, Gomes J. The effect of lovastatin on oxidative stress and antioxidant enzymes in hydrogen peroxide intoxicated rat. Food Chem Toxicol. 2011;49(4):898-902.

18. Jiang P, Mukthavaram R, Chao Y, Nomura N, Bharati IS, Fogal V, et al. In vitro and in vivo anticancer effects of mevalonate pathway modulation on human cancer cells. Br J Cancer. 2014;111(8):1562-71.

19. Henninger C, Huelsenbeck J, Huelsenbeck S, Grösch S, Schad A, Lackner KJ, et al. The lipid lowering drug lovastatin protects against doxorubicin-induced hepatotoxicity. Toxicol Applied Pharmacol. 2012 May 15;261(1):66-73. 
20. Huelsenbeck J, Henninger C, Schad A, Lackner KJ, Kaina B, Fritz G. Inhibition of Rac1 signaling by lovastatin protects against anthracycline-induced cardiac toxicity. Cell Death Dis. 2011;2(8):e190.

21. Bopp A, Wartlick F, Henninger C, Kaina B, Fritz G. Rac1 modulates acute and subacute genotoxininduced hepatic stress responses, fibrosis and liver aging. Cell Death Dis. 2013 Mar;4(3):e558.

22. Grigorian A, O’Brien CB. Hepatotoxicity Secondary to Chemotherapy. J Clin Transl Hepatol. 2014;2(2):95-106.

23. Desai VG, Herman EH, Moland CL, Branham WS, Lewis SM, Davis KJ, et al. Development of doxorubicin-induced chronic cardiotoxicity in the B6C3F1 mouse model. Toxicol Appl Pharmacol. 2013 Jan 1;266(1):109-21.

24. Octavia Y, Tocchetti CG, Gabrielson KL, Janssens S, Crijns HJ, Moens AL. Doxorubicin-induced cardiomyopathy: From molecular mechanisms to therapeutic strategies. J Mol Cell Cardiol. 2012;52(6):1213-25.

25. 2Kalender Y, Yel M, Kalender S. Doxorubicin hepatotoxicity and hepatic free radical metabolism in rats. Toxicology. 2005;209(1):39-45.

26. Tallarico D, Rizzo V, Maio F di, Petretto F, Bianco G, Placanica G, et al. Myocardial Cytoprotection by
Trimetazidine Against Anthracycline-Induced Cardiotoxicity in Anticancer Chemotherapy. Angiology. 2003;54(2):219-27.

27. Osmak M. Statins and cancer: Current and future prospects. Cancer Lett. 2012;324(1):1-12.

28. Ramanjaneyulu SV, Trivedi PP, Kushwaha S, Vikram A, Jena GB. Protective role of atorvastatin against doxorubicin-induced cardiotoxicity and testicular toxicity in mice. J Physiol Biochem. 2013 Sep $1 ; 69(3): 513-25$.

29. Jose J. Statins and its hepatic effects: Newer data, implications, and changing recommendations. J Pharmacy Bioallied Sci. 2016 Jan;8(1):23.

30. 30. Sikka P, Saxena KK, Kapoor S. Statin Hepatotoxicity: Is it a Real Concern? Heart Views. 2011;12(3):104-6.

Cite this article as: Sikandar A, Farhat K, Hamza A. Evaluation and comparison of the hepatoprotective effects of trimetazidine and lovastatin against doxorubicin-induced hepatotoxicity. Int J Basic Clin Pharmacol 2019;8:693-9. 